\title{
Chemical recognition in terahertz time-domain spectroscopy and imaging
}

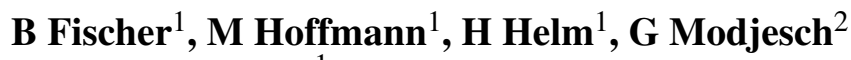 \\ and $P$ Uhd Jepsen ${ }^{1}$ \\ ${ }^{1}$ FMF and Department of Molecular and Optical Physics, Albert-Ludwigs-Universität, \\ D-79104 Freiburg, Germany \\ ${ }^{2}$ Pharmaceutical Institute, Albert-Ludwigs-Universität, D-79104 Freiburg, Germany
}

Received 21 January 2005

Published 8 June 2005

Online at stacks.iop.org/SST/20/S246

\begin{abstract}
In this paper, we present an overview of chemical recognition with ultrashort $\mathrm{THz}$ pulses. We describe the experimental technique and demonstrate how signals for chemical recognition of substances in sealed containers can be obtained, based on the broadband absorption spectra of the substances. We then discuss chemical recognition in combination with $\mathrm{THz}$ imaging and show that certain groups of biological substances may give rise to characteristic recognition signals. Finally, we explore the power of numerical prediction of absorption spectra of molecular crystals and illuminate some of the challenges facing state-of-the-art computational chemistry software.
\end{abstract}

\section{Introduction}

The recently developed technology of generating and detecting ultrashort and coherent electromagnetic pulses in the veryfar-infrared region [1] opens a practical avenue to chemical imaging with very-far-infrared radiation (FIR). The short duration of the pulses results in a bandwidth covering the region from 0.1 to $5 \mathrm{THz}$ of the electromagnetic spectrum. The broadband spectral nature of these pulses permits recording of the dielectric function (absorption coefficient and index of refraction) from the modification of the shape of an electromagnetic pulse transmitted through the sample [2]. This concept uses the coherent nature of the source to directly trace the electric field of the electromagnetic pulse (rather than its intensity). It also exploits the subpicosecond pulse length to implement time-gated detection, thereby eliminating thermal blackbody noise at these frequencies. The latter feature enables us to operate source, sample and detector at ambient temperature, a great advantage in practical applications.

In this paper, we will give an overview of chemical recognition with ultrashort $\mathrm{THz}$ pulses. Section 2 describes the experimental technique and demonstrates how signals for chemical recognition of substances in sealed containers can be obtained. Section 3 discusses a practical implementation of spatial imaging combined with chemical recognition. Section 4 discusses the possibility of obtaining characteristic recognition signals from biological substances. In section 5 , we explore the power of numerical prediction of absorption spectra of molecular crystals and illuminates some of the challenges facing state-of-the-art computational chemistry software.

In order to observe distinct spectral features in the farinfrared absorption spectrum of a substance some sort of longrange order is required. Substances in the condensed phase are held together by either ionic, covalent or electrostatic forces, and therefore the lowest frequency modes will be associated with intermolecular motion. A medium with long-range ordering of its molecular constituents can support phonon-like intermolecular modes at discrete frequency bands, whereas an amorphous medium will display a continuum of strongly damped intermolecular vibrational modes. At higher frequencies, where intramolecular modes are active, this picture is no longer valid. Here, the vibrational modes of the isolated molecules are found, albeit influenced by the surroundings.

Although THz spectroscopy has been proposed to be able to identify and investigate a wide range of substances, we will, for the reason given above, focus our attention on compounds with crystalline structure. Since the mass of the unit cell must be high and the intermolecular forces must be small in order to move vibrational frequencies into the terahertz frequency regime, we will further restrict our discussion to hydrogenbonded organic crystals. 
Chemical recognition in terahertz time-domain spectroscopy and imaging

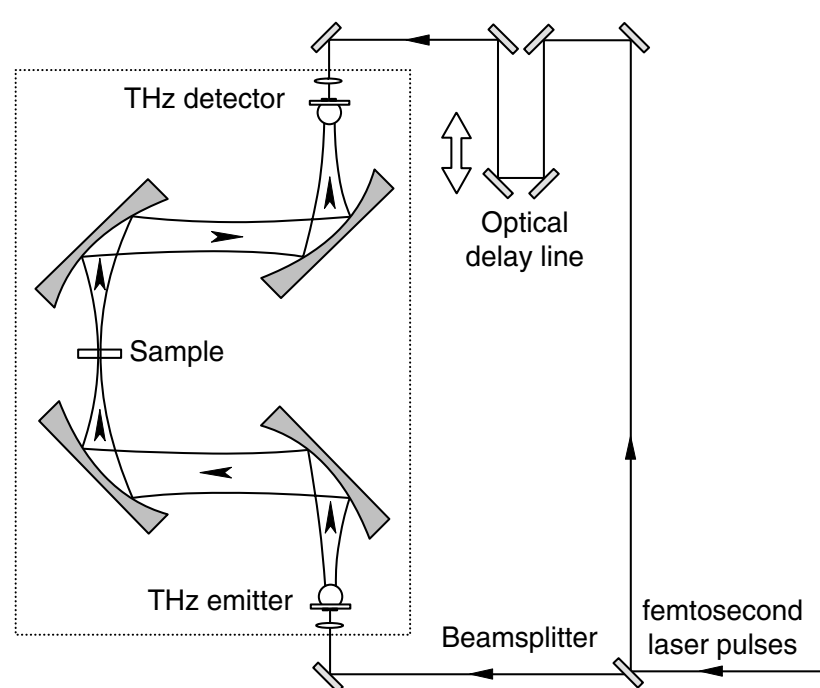

Figure 1. Experimental set-up for chemical recognition of a sample using far-infrared radiation in the transmission mode.

\section{Chemical sensitivity}

To demonstrate the concept of chemical recognition in the $\mathrm{THz}$ frequency domain [3-5], we investigated the pulse modification caused by selected sealed samples using the setup shown in figure 1 . This terahertz time-domain spectrometer uses photoconductive switches for generation and detection of the far-infrared light [6]. The switches are operated with optical $(800 \mathrm{~nm})$ laser pulses of $15 \mathrm{fs}$ duration. This instrument allows us to record the absorption coefficient and index of refraction of samples with a spectral resolution of $0.5 \mathrm{~cm}^{-1}$. This is achieved by tracing the temporal shape of the electric field with sample $E_{\mathrm{S}}(t)$ and without sample $E_{\mathrm{r}}(t)$, where $t$ is the optical delay time of the gating laser pulse and the farinfrared pulse at the detector. The ratio of these fields in the frequency domain $v$

$$
\frac{E_{\mathrm{s}}(v)}{E_{\mathrm{r}}(v)}=\frac{4 n}{(n+1)^{2}} \exp [-\alpha d / 2+\mathrm{i} 2 \pi v(n-1) d / c]
$$

contains the absorption coefficient $\alpha(v)$ and index of refraction $n(v)$ of the sample. The sample thickness is $d$ and $c$ is the speed of light in vacuum.

Two typical traces for $E_{\mathrm{r}}(t)$ and $E_{\mathrm{S}}(t)$, transmitted through an empty envelope and an envelope containing a small amount of lactose powder, are shown in figure 2. The modification of the pulse shape due to the presence of an infrared active compound in the envelope is apparent from the difference in electric field traces. The pulse is delayed and attenuated; both are measures of the dispersion and the absorption by the sample. The spectral key is contained in the pronounced ringing of the sample trace at later times. This ringing is due to the free induction decay of the coherently excited sample [7] which appears as constructive interference in the direction of propagation of the FIR beam.

Figure 3 gives typical spectra of the product $\alpha d$ obtained for several polycrystalline substances sealed in bags made of polyethylene. The absorption spectrum of the empty bag is given as the bottom trace. It is smooth and practically featureless in the low-frequency range. The spectra of lactose, cocaine and morphine show distinct and different features in

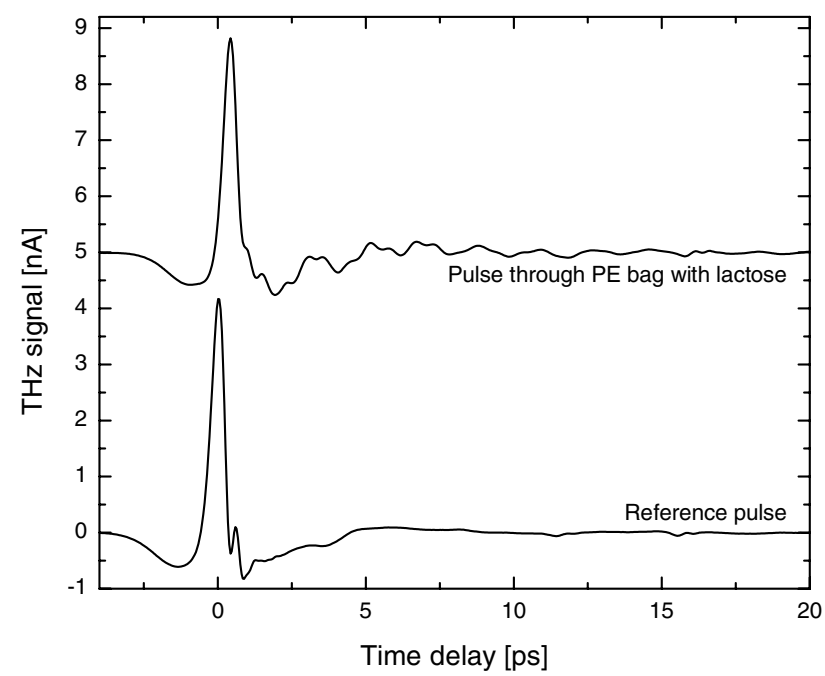

Figure 2. The temporal profile of the electric field strength, here represented as the induced photocurrent in the detector, of the FIR pulse transmitted through an envelope containing lactose and through an empty envelope. The sample trace is offset vertically by $5 \mathrm{nA}$ for better visibility of the traces.

this spectral range. Practically identical spectral signatures are obtained when the samples are enclosed in paper envelopes. A molecular dynamics assignment of the individual resonances in this very-low-frequency range will remain a challenging task, but is irrelevant for the suitability of these spectral fingerprints as a key in chemical recognition.

The identity and purity of the drugs used, cocaine- $\mathrm{HCl}$ (Merck) and morphine-HCl (Synopharm), are according to the prescriptions of The European Pharmacopoeia [15]. The other samples were $\alpha$-lactose monohydrate (glucose free, $4 \% \beta$-anomer content), sucrose (purity $>99.5 \%$ ), aspirin (acetylsalicylic acid, purity $>99.5 \%$ ) and D-tartaric acid (purity $>99 \%$ ).

The course grained samples $(10-100 \mathrm{mg})$ were placed in polyethylene (LDPE) bags with single wall thickness of $55 \mu \mathrm{m}$. The filled bags (thickness $\approx 0.5 \mathrm{~mm}$ ) were placed at the position labelled in figure 1 , shaded by a copper plate with an aperture of $8 \mathrm{~mm}$ diameter. The aperture is filled by the lateral profile of the far-infrared light pulse. The samples were at ambient temperature $(\approx 298 \mathrm{~K})$ when recording the spectra.

No specific precautions were taken to prepare the samples in an optically flat manner when recording the traces shown in figures 2 and 3. To our knowledge no spectral studies have previously been reported for $\alpha$-lactose, cocaine and morphine in this frequency range to which our data could be compared. It is interesting to note that the recently published spectrum of $\beta$-lactose [16] differs significantly from the spectrum of the $\alpha$-anomer used in this work. Some reasons for this difference are that the two anomers differ in the spatial arrangement of atoms and that the $\beta$-anomer in contrast to the $\alpha$-anomer crystallizes without water molecules in the unit cell structure.

Several technical aspects and limitations of our technology need to be mentioned. The typical energy of a single far-infrared pulse in our spectrometer is at the level of $10^{-17} \mathrm{~J}$. Since the spectrometer is operated at a repetition rate of $10^{8} \mathrm{~s}^{-1}$, the sample is exposed to a radiation power of a few $\mathrm{nW}$, a level well below the radiation level experienced by the 

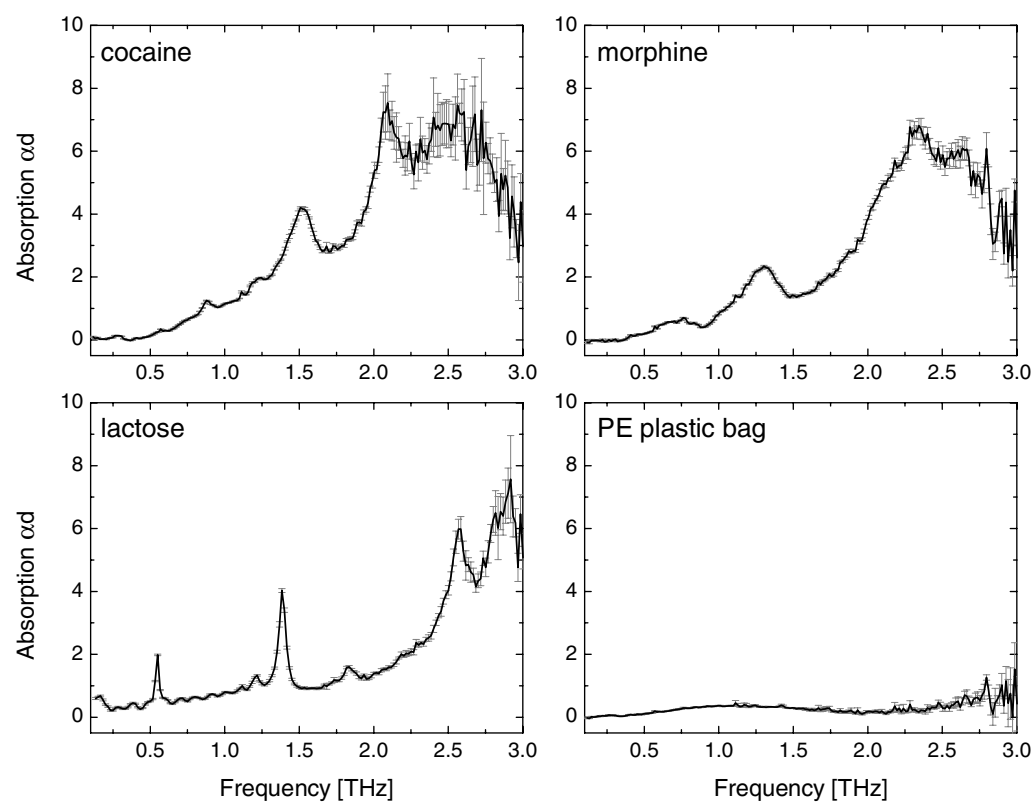

Figure 3. Frequency spectra of the absorption recorded for the empty envelope and identical envelopes containing small amounts of $\alpha$-lactose, cocaine and morphine. The error bars represent one standard deviation from the mean of typically ten measurements.

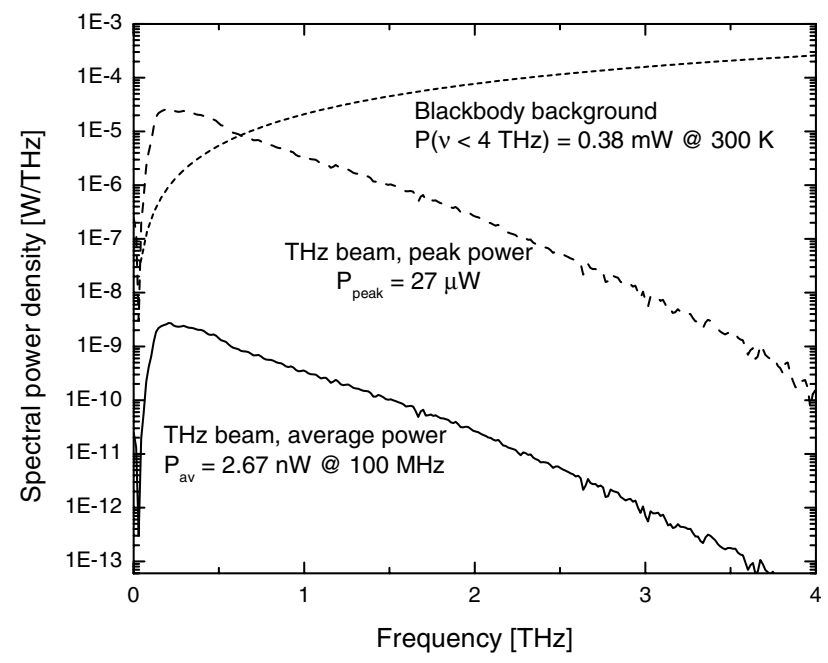

Figure 4. Comparison of blackbody emission from an ideal black surface with $1 \mathrm{~cm}^{2}$ area and the average power of our $\mathrm{THz}$ beam and peak power of the ultrashort $\mathrm{THz}$ pulses.

sample from the thermal background. Even the peak power (taking as pulse width a value of $0.5 \mathrm{ps}$ ) is only $27 \mu \mathrm{W}$. These estimates are quantitatively illustrated in figure 4 , where the spectral power density of the thermal background radiation emitted from a $1 \mathrm{~cm}^{2}$ area at $300 \mathrm{~K}$ is compared to the average and peak spectral power densities experienced by a sample with an area of $1 \mathrm{~cm}^{2}$ in our spectrometer. The radiation load experienced by a sample from the thermal background is several orders of magnitude larger than the average power incident on the sample from the spectrometer. Besides being a non-invasive, contact-free technique, THz-TDS is therefore also quite harmless.

When implemented in the transmission mode, a natural limitation of our approach occurs in optically thick samples. Our current dynamic range for values of $\alpha d$ is from 0.05 to 20 , the lower limit providing ample room for technical improvement. The upper limit for $\alpha d$ is more fundamental, and is linked tightly to the frequency-dependent signal-tonoise ratio of the spectrometer. The limitation in penetrating optically thick samples with a signal sufficient for pulse characterization can be circumvented by operating in reflection geometry [8].

\section{Chemical recognition in terahertz imaging}

It is tempting to explore further possible applications of this technology. The potential of technical imaging in the veryfar-infrared (FIR) range has been considered as early as 1975 . Owing to the lack of easy-to-use sources at that time this topic had not been pursued past an initial proof of principle [9]. Recent advancements in ultrashort electromagnetic pulse generation has revived this subject $[10,11]$. In particular, electro-optical techniques are now capable of upconverting an FIR radiation field pattern into the visible domain [12, 13], permitting live viewing of the spatial and temporal distributions of the electric field strength. Very recently, distinction between different types of biological material in pulsed $\mathrm{THz}$ imaging [17], as well as detection of specific chemicals in scanning continuous-wave THz imaging [18, 19], was demonstrated.

In the following, we will demonstrate that $\mathrm{THz}$ imaging combined with a straightforward THz-TDS analysis of the transmitted $\mathrm{THz}$ pulses allows contact-free and reliable recognition of drugs and other chemicals hidden in containers. The applied recognition algorithm is simple and very fast, and scales linearly with the number of chemical substances in the reference database.

In figure 5(a) a photograph of a sample, consisting of four pellets glued to a piece of paper, is shown. Each pellet contains $60 \mathrm{mg}$ of either lactose, aspirin, sucrose or tartaric 


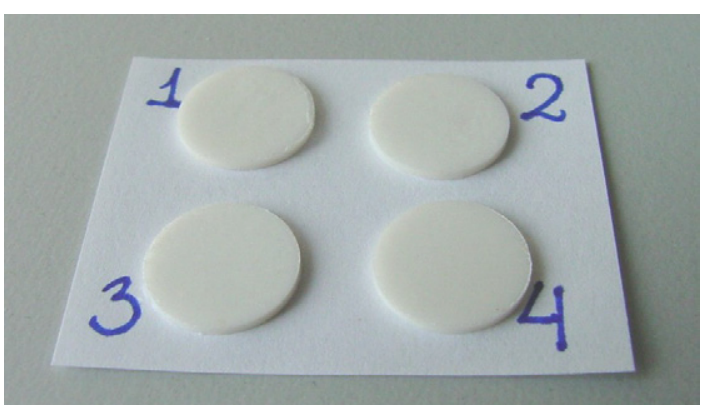

(a)

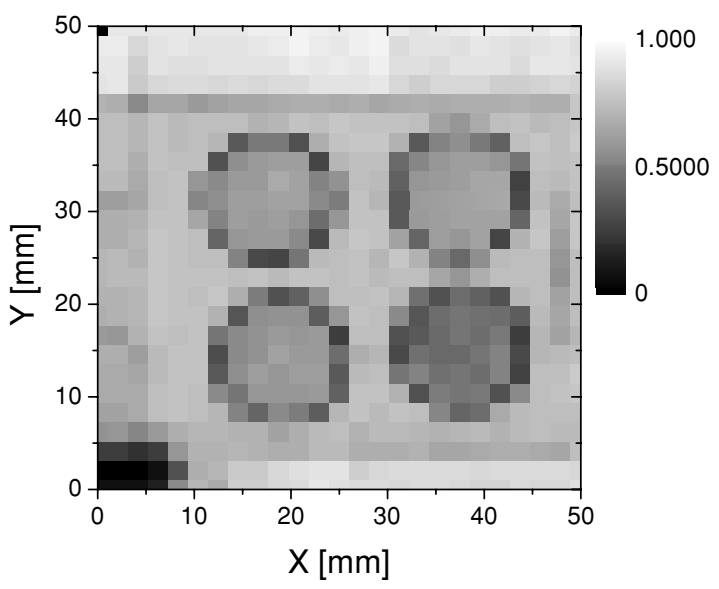

(b)

Figure 5. (a) Visible image of sample with four pellets containing different chemicals and $(b)$ an image of the $\mathrm{THz}$ transmission amplitude.

acid, mixed and pressed with $80 \mathrm{mg}$ of polyethylene powder for mechanical stability.

We record the full THz pulse shape (truncated 5 ps before the main pulse and with a length of $70 \mathrm{ps)} \mathrm{transmitted} \mathrm{through}$ the sample. We use a $25 \times 25$ pixel grid, with a grid spacing of $2 \mathrm{~mm}$. THz images can then be formed by different methods, as will be discussed below.

In figure $5(b)$ the $\mathrm{THz}$ transmission image of the sample is shown, obtained by recording the peak amplitude of the $\mathrm{THz}$ pulse after transmission through the sample, which is mounted on a $1 \mathrm{~cm}$ thick styrofoam plate and covered with a second sheet of paper during the measurements. The four pellets are clearly visible in the $\mathrm{THz}$ image, but no distinction between the chemicals is possible by this simple imaging technique.

In order to identify and distinguish the chemicals in the sample, we define a recognition coefficient $R$ proportional to the height of a spectral feature with respect to its baseline,

$$
R=\alpha\left(v_{2}\right)-\frac{1}{2}\left(\alpha\left(v_{1}\right)+\alpha\left(v_{3}\right)\right),
$$

where $\alpha$ is the absorption coefficient defined in equation (1). If more than one clear spectral feature is present in the absorption spectrum then $R$ may be taken as the sum or product of several peak heights, thereby increasing the specificity. For samples of unknown thickness or irregular shape only the product $\alpha d$ is measured. In this situation, the product of absorption and sample thickness can be used in equation (2), as shown below.

The absorption spectra of lactose, aspirin, sucrose and tartaric acid shown in figure 6 are extracted from the $\mathrm{THz}$ pulses transmitted through the relevant regions of the sample. The solid lines show the absorption averaged over the pellet areas (20-30 pixels), with vertical bars indicating the corresponding standard deviation. This standard deviation is significantly larger than that in figure 3 due to spatial inhomogeneity of the sample. The lower absorption curve in each panel shows the background absorption of the packing material. The standard deviation is indicated for an average over 30 pixels. The vertical lines indicate the frequencies chosen for chemical recognition.

In figure 7 maps of recognition coefficient $R$ for the four chemicals in the sample are shown, using the frequencies indicated in figure 6. For aspirin and tartaric acid we use one peak for recognition, and for lactose and sucrose the sum of two peaks is used.

In spite of its simplicity, the recognition strategy presented here is clearly capable of identifying the four different chemicals contained in the sample. Due to the prominent spectral features in the absorption spectra of lactose and tartaric acid, the signal-to-noise ratio of the recognition signals for those substances is strong and clear. In spite of the weak spectral features and relatively large background absorption of aspirin and sucrose the recognition strategy is still successful, although with slightly lower signal-to-noise ratio.

Within the limits of the dynamical range of the spectrometer, the recognition coefficient is proportional to the concentration of the chemical. With the proper calibration, this strategy can therefore also be used to determine the concentration of the chemical. This capability has been demonstrated in experiments with quasi-continuous-wave $\mathrm{THz}$ radiation by Kawase and co-workers [18, 19]. We note that owing to the relatively broad line shapes in the $\mathrm{THz}$ absorption spectra, chemical recognition with $\mathrm{THz}$ radiation will probably find its best applications in situations where a known and limited range of possible substances may or may not be present in a sample.

\section{Spectral signatures of biomolecules}

Having seen that polycrystalline samples of organic molecules offer specific responses to $\mathrm{THz}$ radiation, it is of interest to investigate if specific responses can be obtained from biological materials. However, since biological systems typically lack a crystalline structure it can be expected that only a limited group of biological materials will possibly show distinct resonance frequencies in the $\mathrm{THz}$ range.

Biopolymers are a group of biologically interesting materials with a natural long-range order. Among the most important biopolymers are carbohydrate energy storage molecules such as cellulose which is one of the most abundant organic compounds in the biosphere, and chitin which is responsible for the structural strength of exoskeletons of insects and crustatea. DNA is another biopolymer of immense importance for all life.

In order to investigate the possibility of finding spectral signatures of biomolecules in the $\mathrm{THz}$ range, we have measured the absorption spectra of cellulose, chitin and a small oligonucleotide. The molecular structures of cellulose and chitin are shown in figure 8 , along with the chemical structure of a small artificial single-stranded oligonucleotide 

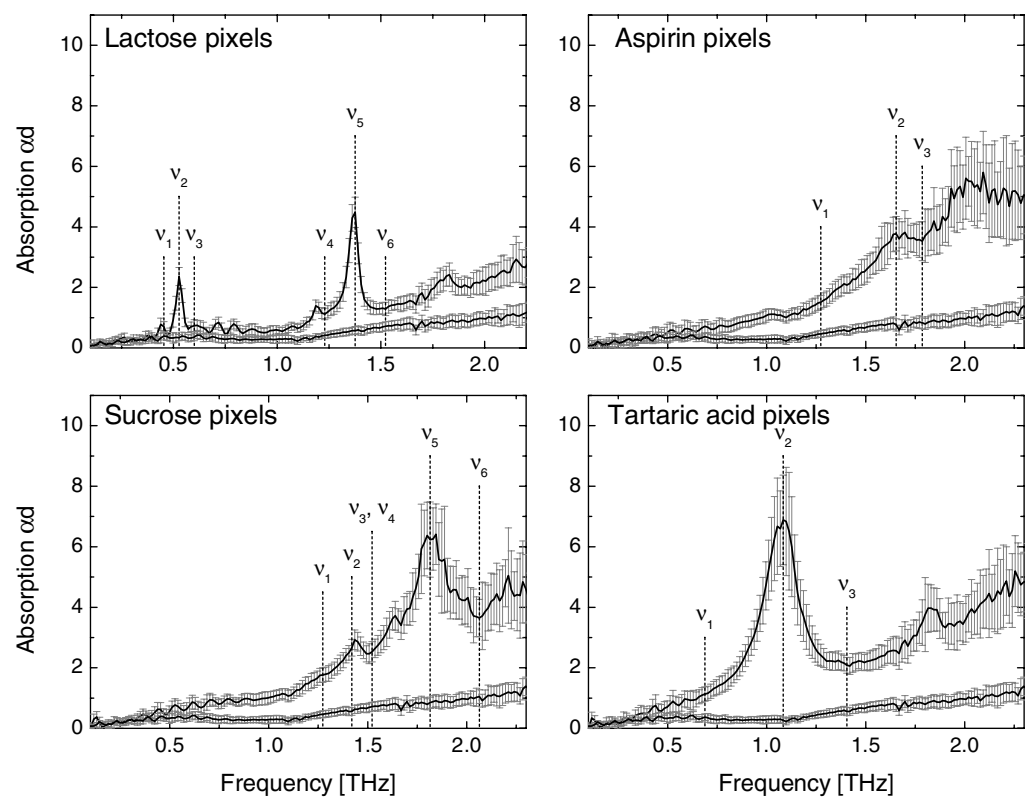

Figure 6. Solid lines show the average absorption of lactose, aspirin, sucrose and tartaric acid in the sample. The lower curve in each panel shows the absorption of the packing material. The error bars represent one standard deviation from the mean of typically $20-30$ measurements. The indicated frequencies are used for chemical recognition.
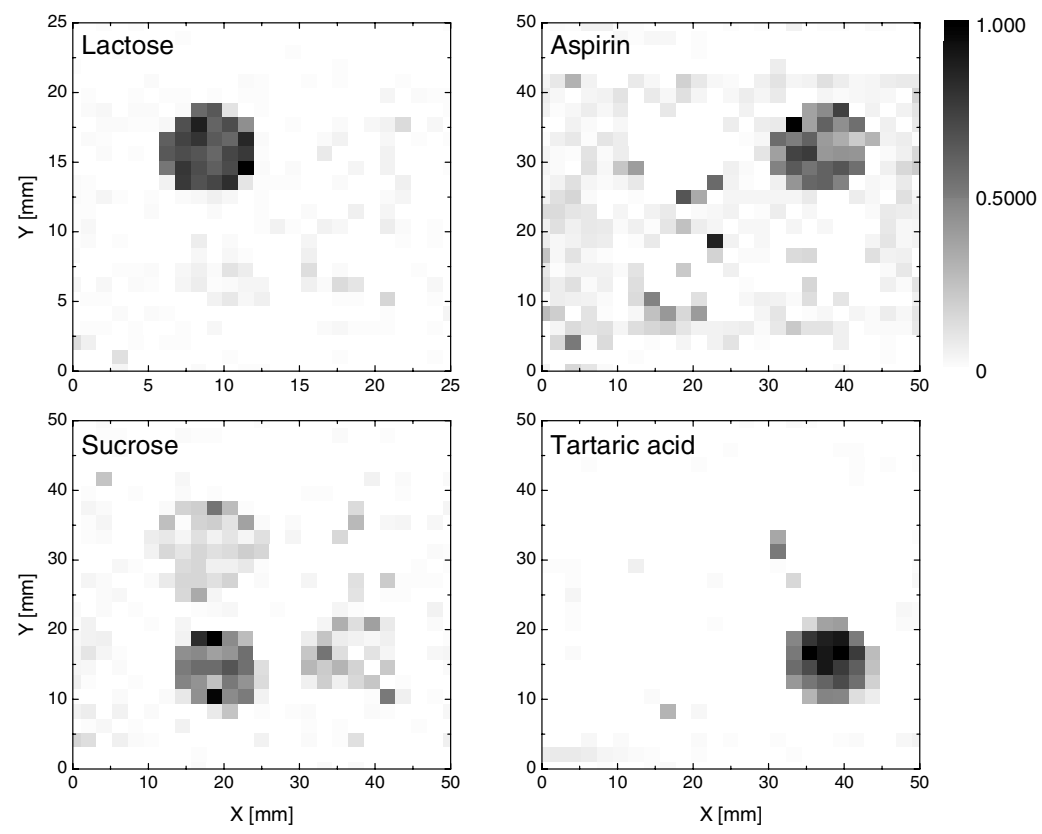

Figure 7. Maps of the recognition coefficient $R$ for the four different chemicals in the sample.

with the base sequence A-T-A-T-A. The absorption spectra of the three substances are shown in figure 9. The upper panel shows the absorption spectra at room temperature and the lower panel shows the corresponding spectra recorded at $10 \mathrm{~K}$. The samples consisted of $115 \mathrm{mg}$ cellulose, $25 \mathrm{mg}$ chitin and less than $1 \mathrm{mg}$ of the oligonucleotide. The chitin and the oligonucleotide material were mixed with polyethylene powder and all samples were pressed to pellets to ensure plane interfaces of the samples. At room temperature, the absorption spectra of all samples are dominated by a monotonously increasing absorption. This absorption slope may be the result of scattering since the particle size in the samples becomes comparable to the wavelength at the highest frequencies. Another contribution to this background absorption may come from amorphous phases present in the samples. The spectral shape resembles closely that of rapidly cooled, and therefore amorphous, sugar melts [5].

When the samples are cooled to $10 \mathrm{~K}$ (lower panel of figure 9), distinct absorption features emerge on top of the broad background absorption. In cellulose, we observe an absorption band centred at $2.15 \mathrm{THz}$ with a FWHM width of $0.35 \mathrm{THz}$. In chitin, we observe a weaker band centred at $1.7 \mathrm{THz}$ with a FWHM width of $0.45 \mathrm{THz}$. The oligonucleotide displays an absorption feature at $2.4 \mathrm{THz}$ with 
Cellulose<smiles>C[C@@H](O)O[C@H]1O[C@H](C)[C@@H](O[C@H]2O[C@H](CO)[C@@H](O)[C@H](O)[C@H]2O)[C@H](O)[C@H]1O</smiles>

Chitin

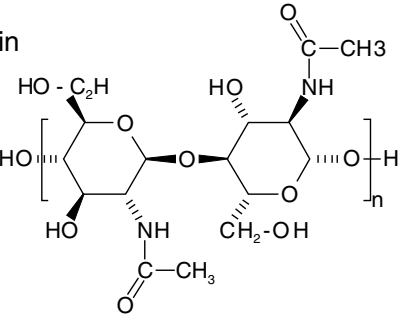

$d A-d T-d A-d T-d A$

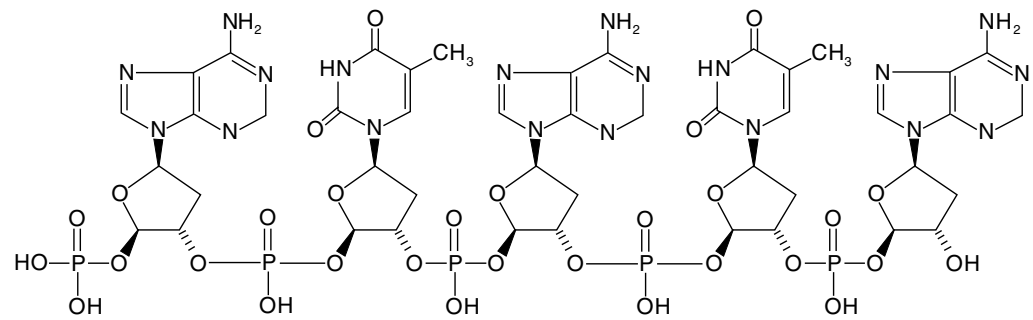

Figure 8. Chemical structures of the biopolymers cellulose, chitin and the single-strand oligonucleotide sequence A-T-A-T-A.

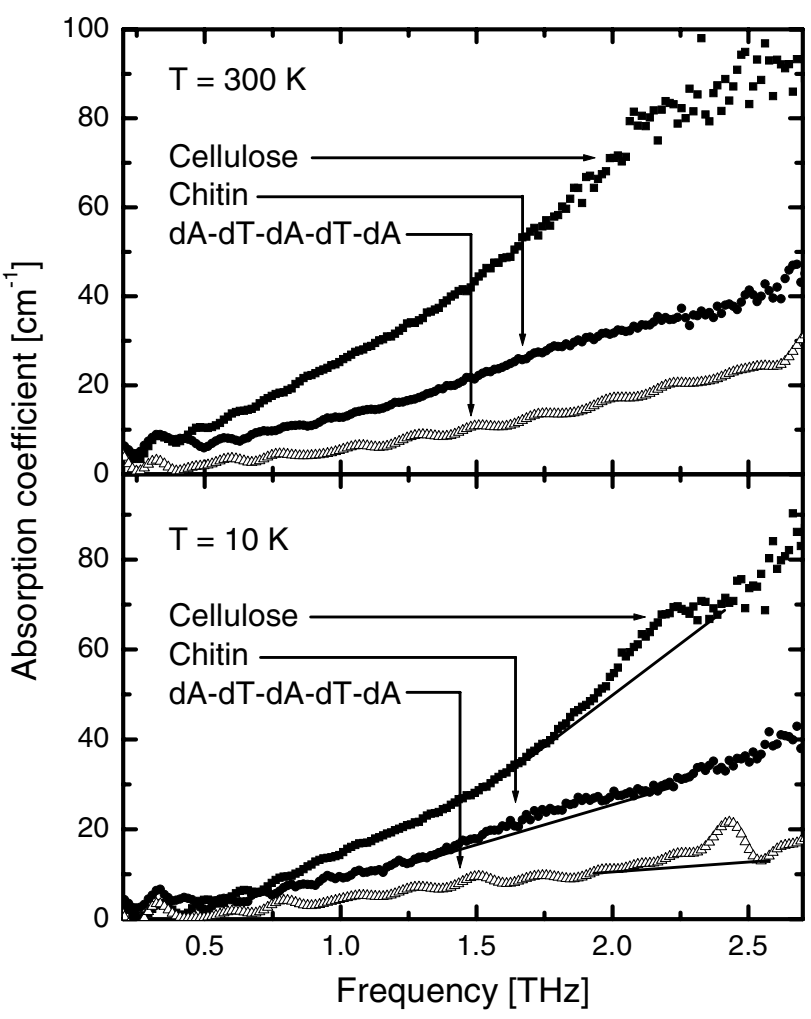

Figure 9. Absorption spectra at room temperature (upper panel) and $10 \mathrm{~K}$ (lower panel) of cellulose, chitin and the oligonucleotide A-T-A-T-A in the frequency range $0.2-2.7 \mathrm{THz}$.

a FWHM width of $0.15 \mathrm{THz}$. For reasons discussed in the next section, a vibrational analysis of the large molecular structures is a very demanding challenge. In the case of cellulose and chitin, we speculate that the observed features may arise from a $1 \mathrm{D}$ phonon-like mode along the backbone of the polymer. The lowering of the resonance frequency in chitin is consistent with the larger mass of the side groups of the chitin backbone compared to cellulose.
The examples shown above demonstrate that it is possible to detect isolated spectral features even in large biomolecular systems. The systems investigated here are all free of water and with a significant fraction of the sample material in a crystalline state. Biomolecular systems in aqueous environments cannot in general be expected to form such macroscopically wellordered phases, and therefore the $\mathrm{THz}$ dielectric properties of such systems are expected to contain no discrete features.

\section{Prediction of far-infrared absorption spectra}

The theoretical approach to the calculation of vibrational modes of a molecular system is based on finding the potential energy surface of the molecule. Although the basic theoretical framework has been known since the early days of quantum mechanics, the practical implementation is still a major computational problem. Hartree-Fock (HF) theory is the simplest approach to find an approximate energy surface of the system. However, the motion of electrons with opposite spin is completely uncorrelated within the HF theory. Correlation between electrons leads to corrections to the energetic surface of the molecule. Especially for the prediction of low-frequency vibrational modes associated with shallow potential energy minima, these correlation corrections are of vital importance. Therefore, higher level theory is needed in order to calculate intermolecular vibrational motion with sufficient accuracy.

Density functional theory (DFT) approaches the inclusion of correlation between electrons by calculation of an energy functional of the electron spin densities and possibly their gradients. The trick of the trade is to define good energy functionals which reproduce known quantities of model systems.

Becke's three-parameter energy functional [20], which is a hybrid of HF exchange terms and DFT exchange and correlation terms, combined with the LYP functional by Lee et al [21] (B3LYP), has proven itself to be a strong method for calculation of molecular properties including vibrational frequencies. The computational cost of this method is low 


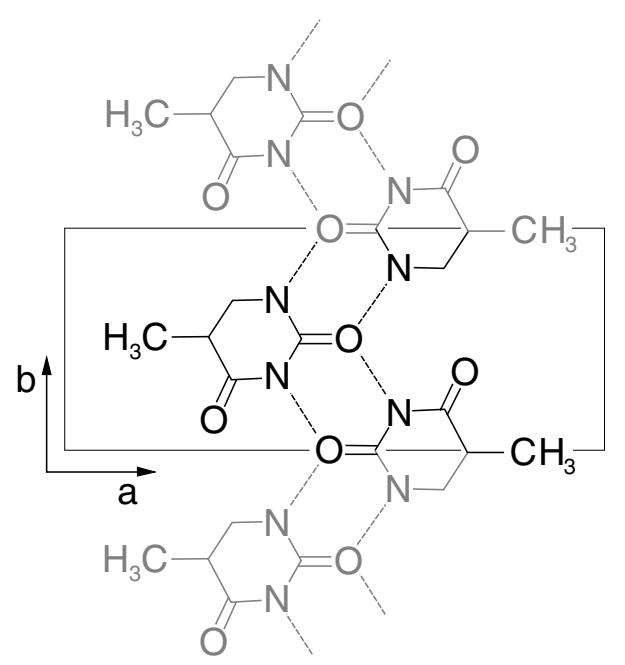

Figure 10. Crystalline and molecular structure of thymine. The boxed area indicates the $a-b$-plane of the unit cell of the crystalline structure. The dashed lines indicate the intermolecular hydrogen bond network.

compared to other correlation methods such as Møller-Plesset (MP) theory or configuration interaction (CI) methods.

In the following, we will present a calculation of the farinfrared vibrational spectrum of a molecular crystal. The DNA base thymine forms a relatively simple hydrogenbonded crystalline structure, as shown in figure 10 [22]. The dashed lines between the thymine molecules indicate the intermolecular hydrogen bonds. The unit cell includes two additional thymines, displaced along the $c$-direction (out of the plane, not shown in the figure). The interaction between the molecules is strong in the $a$-direction within the crystal plane shown in figure 10. The interaction between the layers in the $c$-direction and between neighbouring rows in the $b$-direction is much weaker. It may therefore be expected that a computation of the vibrational frequencies of the structure shown in figure 10 will be able to reproduce at least the major features of the measured absorption spectrum of thymine crystals.

In figure 11, we show the result of DFT calculations of thymine molecular structures. The figure shows the vibrational spectrum for structures containing between 1 and 8 thymine molecules, aligned as shown in figure 10. The calculations were performed with the computational chemistry software package Gaussian 03 [23]. We used the B3LYP theory with the 6-31G basis set. The upper trace of figure 11 is the measured absorption spectrum of polycrystalline thymine powder at a temperature of $10 \mathrm{~K}$. The line width of the calculated vibrational modes is given by the calculation. We have simply assumed a line width of $3 \mathrm{~cm}^{-1}$ for all modes. The relative strengths of the modes are shown as calculated. The geometry of each of the structures was optimized at the same theory level and with the same basis set before the frequency calculation.

From the progression of the calculated spectra, it is clear that the vibrational properties of a single molecule is in no way related to the observed spectrum. As more molecules are added to the structure a stable band of frequencies in the region around $80 \mathrm{~cm}^{-1}$ develops, closely resembling the absorption bands in the observed spectrum. Although the computation was limited to structures containing up to eight thymine

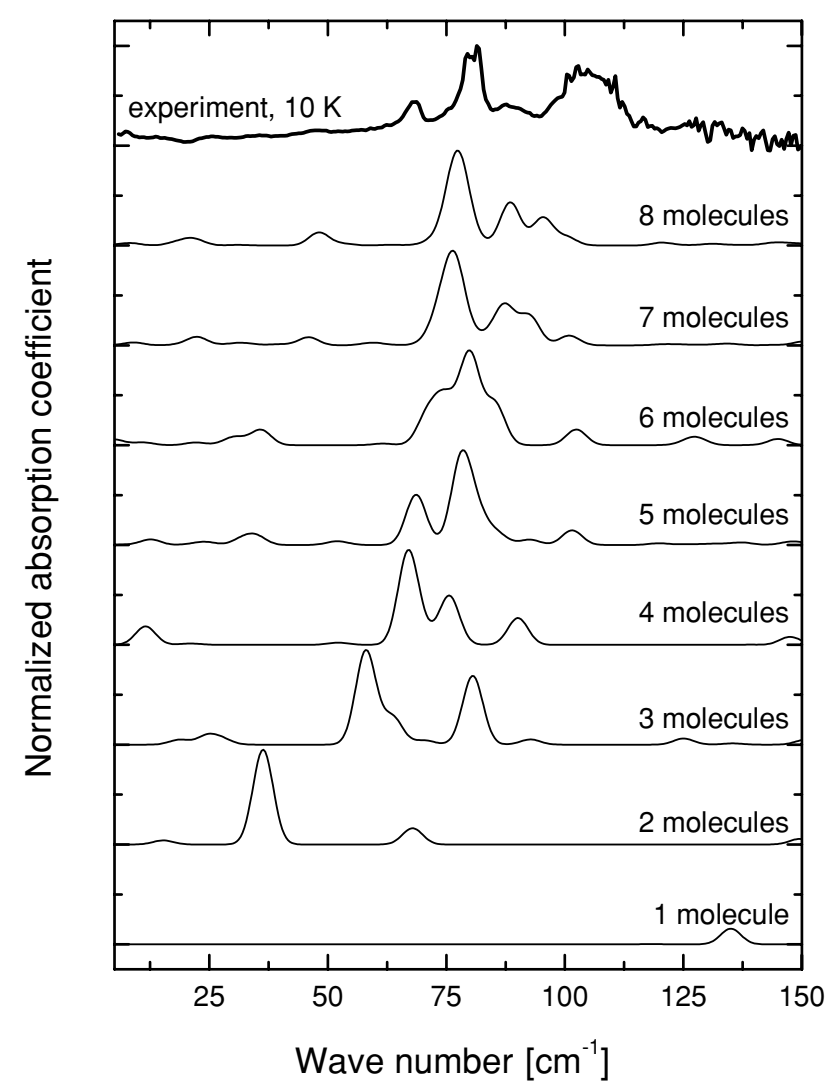

Figure 11. Predicted vibrational spectra of thymine clusters of increasing size compared to the measured absorption spectrum (upper trace) of polycrystalline thymine.

molecules, it is apparent that the computation describes the observed spectral features. A normal mode analysis shows that the strongest modes in the $80 \mathrm{~cm}^{-1}$ band are out-of-plane and in-plane intermolecular bending modes, which for a full crystal structure correspond to various phonon modes.

The relatively good agreement between $a b$ initio theory and experiment is in the example shown here strongly facilitated by the simple arrangement of molecules in the crystal structure. The arrangement allows us to reduce the calculation to a structure which to a good approximation grows in only one dimension. This is, unfortunately, not the situation in most molecular crystals. In the general case, intermolecular interactions are of similar strength in all three dimensions. In such a case, the cluster of molecules included in the calculation must grow in all three dimensions in order to observe the lattice vibrations. The CPU time needed for the calculation presented in figure 11 scales as $n^{2.75}$, where $n$ is the number of thymine molecules. For a full three-dimensional cluster formation, it can therefore be expected that the CPU cost grows at least as $d^{8}$, where $d$ is the linear dimension of the cluster. This is of course far too expensive in terms of CPU power to be feasible, so clearly other methods are needed for the numerical prediction of far-infrared absorption spectra of molecular crystals.

An obvious direction of progress will be to include periodic boundary conditions in DFT calculations and develop efficient algorithms for calculation of phonon band structures of molecular crystals.

Alternatively, molecular mechanics calculations with adapted force fields have in some situations shown good 
agreement with experimental spectra of crystalline organic compounds $[24,25]$. The success of this approach depends strongly on an adaption of the force field parameters to specific molecule in order to reproduce the experimental vibrational spectrum. Thus, in spite of the good agreement with experiment that can be obtained, this method has limited predictive strength for molecular systems to which the force field has not been adapted. On the other hand, if a force field valid for a broad range of compounds can be found, then this method holds the potential for prediction of far-infrared vibrational spectra.

\section{Conclusions}

In conclusion, we have demonstrated how spatially resolved THz-TDS can be used as a general method for detection of chemicals hidden in sealed containers transparent to $\mathrm{THz}$ radiation. The contrast mechanism that allows a distinction between the different chemicals is the highly specific free induction decay signal which is emitted coherently by the sample subsequent to excitation of collective vibrational modes of the crystal lattice by the ultrashort, broadband $\mathrm{THz}$ pulse. In the frequency domain, the FID signal corresponds to transitions between vibrational states of the crystalline compound. In the $\mathrm{THz}$ range such transitions mainly arise from lattice modes, which are specific to the crystalline structure as well as the molecular structure, and hence form a unique fingerprint of the substance. Contemporary computational chemistry can assist to understand the origin of these modes, and in the future the far-infrared spectra of compounds may even be accurately predicted by numerical calculations. A key issue is the inclusion of periodic boundary conditions in the calculation of vibrational frequencies.

Irrespective of the capability to predict far-infrared spectra, the chemical recognition principles discussed here can extract the chemical information in a simple and fast way. More sophisticated data analysis based on pattern recognition will significantly improve the detection capability. We believe that this approach to imaging in the far-infrared holds great promise in chemical, pharmaceutical and also in certain biological applications where a specific sample response in the far infrared can be expected.

\section{Acknowledgments}

This research was partially supported by BMBF and the EU project THz-BRIDGE under contract number QLK4CT-2000-00129 and by project start-up funds provided by the Albert-Ludwigs-University under grant number ZEE20030131.

\section{References}

[1] van Exter M, Fattinger C and Grischkowsky D 1989 Terahertz time-domain spectroscopy of water vapor Opt. Lett. 14 1128-30

[2] Grischkowsky D, Keiding S R and van Exter M 1990 Far-infrared time-domain spectroscopy with terahertz beams of dielectric and semiconductors J. Opt. Soc. Am. 7 2006-15
[3] Söderholm S, Roos Y H, Meinander N and Steinby K 2000 Temperature dependence of the Raman spectra of amorphous glucose in the glassy and supercooled liquid states J. Raman Spectrosc. 31 995-1003

[4] Young L, Weng S, Ferraro J R and Wu J 2001 Far infrared study of some mono- and disaccharides Vib. Spectrosc. 25 $57-62$

[5] Walther M, Fischer B M and Jepsen P U 2003 Noncovalent intermolecular forces in polycrystalline and amorphous saccharides in the far infrared Chem. Phys. 288 269-76

[6] Walther M, Fischer B M, Schall M, Helm H and Jepsen P U 2000 Far-infrared vibrational spectra of all-trans 9-cis and 13-cis retinal measured by THz time-domain spectroscopy Chem. Phys. Lett. 332 389-95

[7] Harde H, Katzenellenbogen N and Grischkowsky D 1994 Terahertz coherent transients from methyl chloride vapor J. Opt. Soc. Am. 11 1018-30

[8] Thrane L, Jacobsen R H, Jepsen P U and Keiding S R 1995 THz-reflection spectroscopy of liquid water Chem. Phys. Lett. 240 330-3

[9] Barker D H, Hodges D T and Hartwick T S 1975 Far infrared imagery Proc. SPIE 67 27-34

[10] Hu B B and Nuss M C 1995 Imaging with terahertz waves Opt. Lett. $201716-8$

[11] Mittleman D 2003 Sensing with Terahertz Radiation (Berlin: Springer) p 85

[12] Jepsen P U, Schall M, Schyja V, Winnewisser C, Helm H and Keiding S R 1996 Detection of high power THz pulses by phase retardation in an electro-optic crystal Ultrafast Processes in Spectroscopy ed Svelto et al (New York: Plenum) pp 645-8

[13] Zhang X-C, Wu Q, Campbell P and Libelo L 1996 New field sensors for subpicosecond electromagnetic pulses Ultrafast Processes in Spectroscopy ed O Svelto et al (New York: Plenum) pp 649-52

[14] Jepsen P U, Winnewisser C, Schall M, Schyja V, Keiding S R and Helm H 1996 Detection of THz pulses by phase retardation in lithium tantalate Phys. Rev. E 53 R3052-4

[15] Europäisches Direktorat für die Qualität von Arzneimitteln (EDQM) des Europarats 2002 The European Pharmacopoeia 4th edn

[16] Upadhya P C, Shen Y C, Davies A G and Linfield E H 2004 Far-infrared vibrational modes of polycrystalline saccharides Vib. Spectrosc. 35 139-43

[17] Ferguson B, Wang S, Gray D, Abbott D and Zhang X-C 2002 Identification of biological tissue using chirped probe $\mathrm{THz}$ imaging Microelectron. J. 33 1043-51

[18] Watanabe Y, Kawase K, Ikari T, Ito H, Ishikawa Y and Minanide H 2003 Component spatial pattern analysis of chemicals using terahertz spectroscopic imaging Appl. Phys. Lett. 83 800-2

[19] Kawase K, Ogawa Y, Watanabe Y and Inoue H 2003 Non-destructive terahertz imaging of illicit drugs using spectral fingerprints Opt. Express 11 2549-54

[20] Becke A D 1993 Density-functional thermochemistry: III. The role of exact exchange J. Chem. Phys. 985648

[21] Lee C, Yang W and Parr R G 1988 Development of the Colle-Salvetti correlation-energy formula into a functional of the electron density Phys. Rev. B 37785

[22] Ozeki K, Sakabe N and Tanaka J 1969 The crystal structure of thymine Acta Cryst. B 25 1038-45

[23] Frisch M J et al 2003 Gaussian 03, Revision B.04 (Pittsburgh PA: Gaussian, Inc.)

[24] Dauchez M, Derreumaux P and Vergoten G 1992 Vibrational molecular force field of model compounds with biologic interest: II. Harmonic dynamics of both anomers of glucose in the crystalline state J. Comp. Chem. 14 263-77

[25] Sekkal M, Legrand P, Vergoten G and Dauchez M 1992 A vibrational molecular force field of model compounds with biological interest: III. Harmonic dynamics of $\alpha$ - and $\beta$-D-galactose in the crystalline state Spectrochim. Acta A 48 959-73 